\title{
Exercise Improves Outcomes of Surgery on Fatty Liver in Mice
}

\author{
A Novel Effect Mediated by the AMPK Pathway
}

\author{
Michael Linecker, MD, ${ }^{*}$ Lukas Frick, MD, ${ }^{*}$ Philipp Kron, MD, ${ }^{*}$ Perparim Limani, MD, PhD, * \\ Patryk Kambakamba, MD, ${ }^{*}$ Christoph Tschuor, MD, PhD, ${ }^{*}$ Magda Langiewicz, PhD, ${ }^{*}$ \\ Ekaterina Kachaylo, PhD, ${ }^{*}$ Yinghua Tian, MD, ${ }^{*}$ Marcel A. Schneider, MD, ${ }^{*}$ Udo Ungethüm, BS, \\ Nicolas Calo, PhD, $\dagger$ Michelangelo Foti, PhD, $\dagger$ Jean-François Dufour, MD, $\ddagger$ Rolf Graf, PhD, * \\ Bostjan Humar, PhD, ${ }^{*}$ and Pierre-Alain Clavien, MD, PhD*
}

\begin{abstract}
Objective: To investigate whether exercise improves outcomes of surgery on fatty liver, and whether pharmacological approaches can substitute exercising programs.

Summary of Background Data: Steatosis is the hepatic manifestation of the metabolic syndrome, and decreases the liver's ability to handle inflammatory stress or to regenerate after tissue loss. Exercise activates adenosine monophosphate-activated kinase (AMPK) and mitigates steatosis; however, its impact on ischemia-reperfusion injury and regeneration is unknown.

Methods: We used a mouse model of simple, diet-induced steatosis and assessed the impact of exercise on metabolic parameters, ischemia-reperfusion injury and regeneration after hepatectomy. The same parameters were evaluated after treatment of mice with the AMPK activator 5-aminoimidazole-4-carboxamide ribonucleotide (AICAR). Mice on a control diet served as age-matched controls.

Results: A 4-week-exercising program reversed steatosis, lowered insulin levels, and improved glucose tolerance. Exercise markedly enhanced the ischemic tolerance and the regenerative capacity of fatty liver. Replacing exercise with AICAR was sufficient to replicate the above benefits. Both exercise and AICAR improved survival after extended hepatectomy in mice challenged with a Western diet, indicating protection from resection-induced liver failure.

Conclusions: Exercise efficiently counteracts the metabolic, ischemic, and regenerative deficits of fatty liver. AICAR acts as an exercise mimetic in settings of fatty liver disease, an important finding given the compliance
\end{abstract}

From the *Swiss HPB and Transplant Center, University Hospital of Zürich, Zürich, Switzerland; †Department of Cellular Physiology and Metabolism, Faculty of Medicine, University of Geneva, Geneva, Switzerland; and $\ddagger$ Hepatology Unit, Department of Clinical Research, University Hospital of Bern, Bern, Switzerland.

Disclosure: $\mathrm{BH}$ and PAC shared senior authorship.

The authors declare no conflicts of interest.

Authors' contributions: ML, LF, PKr, PL, PKa, CT, ML, EK, YT, MAS, UU, and NC contributed in the acquisition of data. BH, ML, MF, and JFD contributed in the analysis and interpretation of data. $\mathrm{BH}$ and $\mathrm{ML}$ contributed in the drafting of the article; $\mathrm{BH}, \mathrm{ML}$, and PAC contributed in the critical revision of the manuscript for important intellectual content. ML and LF contributed in the statistical analysis; $\mathrm{BH}, \mathrm{PAC}$ obtained funding; $\mathrm{BH}$ contributed in the study concept and design; administrative, technical, or material support was done by JFD; study supervision was done by $\mathrm{BH}, \mathrm{RG}$, and PAC.

This study was supported by the LGID (Liver and Gastrointestinal Disease) Foundation, Zurich, Switzerland, and through Sinergia Grants from the Swiss National Science Foundation (CRSII3_141798/1 and CRSII3_160717/1).

Supplemental digital content is available for this article. Direct URL citations appear in the printed text and are provided in the HTML and PDF versions of this article on the journal's Web site (www.annalsofsurgery.com).

Reprints: Pierre-Alain Clavien, MD, PhD, Department of Surgery and Transplantation, University Hospital Zurich, Rämistrasse 100, CH-8091 Zürich, Switzerland. E-mail: clavien@access.uzh.ch.

Copyright (C) 2018 Wolters Kluwer Health, Inc. All rights reserved.

ISSN: 0003-4932/16/XXXX-0001

DOI: $10.1097 /$ SLA.0000000000002904 issues associated with exercise. Exercising, or its substitution through AICAR, may provide a feasible strategy to negate the hepatic consequences of energy-rich diet, and has the potential to extend the application of liver surgery if confirmed in humans.

Keywords: AMPK activation, ischemia-reperfusion injury, liver resection, nonalcoholic fatty liver disease, physical activity, treadmill running

(Ann Surg 2018;xx:xxx-xxx)

$M$ etabolic diseases associated with Western-style diet are continuing to spread. ${ }^{1}$ The liver as the central metabolic organ is a primary target of nutritional overload. The consumption of energy-rich diet is strongly associated with a progressive cascade of diseases, typically starting with nonalcoholic fatty liver disease (NAFLD) that may progress to nonalcoholic steatohepatitits (NASH) and even liver cancer.,3

Exercise is well known to mitigate the negative consequences of energy-rich diet by re-directing metabolism towards the consumption of fat stores. Exercise - also in the absence of body weight loss can reduce hepatic steatosis and improve insulin sensitivity. ${ }^{4-6}$ More so, exercising individuals display increased $\beta$-oxidation with improved insulin sensitivity even when maintaining a high caloric intake. ${ }^{7}$ In experimental models of fatty liver disease, the effects of exercise on lipid oxidation, lipogenesis, and glucose tolerance are superior over those of dietary restriction. ${ }^{2,8}$ The molecular changes occurring under exercise may hence point to pharmaceutical targets against metabolic liver disease.

Exercise is believed to exert its beneficial effects via metabolic reprogramming. The adenosine monophosphate-activated kinase (AMPK) in its role as an intracellular fuel sensor is a central player in these processes. Drops in adenosine triphosphate: adenosine diphosphate/ adenosine monophosphate (ATP:ADP/AMP) ratios during exercise activate AMPK to respond to energy deprivation. In turn, AMPK suppresses lipogenesis and stimulates $\beta$-oxidation by modulating a number of metabolic pathways, such as the dampening of growth-related mechanistic target of rapamycin (mTOR) activities or the promotion of catabolic peroxisome proliferator-activated receptor alpha (PPAR $\alpha)$ action. $^{2}$

Given the obvious benefits of exercise in metabolic liver disease, we sought to determine whether similar gains for liver health can be achieved through an AMPK activator. Indeed, nutritional overload does not only disturb the metabolic function of liver as exemplified through insulin resistance and glucose intolerance. ${ }^{1,2,4,6}$ Fatty liver also displays a markedly reduced ability to tolerate ischemic injury or to regenerate after tissue loss. ${ }^{9}$ The impact of exercise on acute liver stress however is unknown. We therefore established a mouse model of diet-induced steatosis, where we were able to replicate the known metabolic benefits of exercising. Using this validated model of steatosis, we went on to compare the effects 
of exercise on steatosis, ischemic tolerance and regenerative capacity with those of a pharmacological AMPK activator.

\section{MATERIAL AND METHODS}

\section{Animals}

All animal experiments were performed in accordance with Swiss Federal Animal Regulations and approved by the Veterinary Office of Zurich. Male C57BL6 mice (Harlan, Horst, the Netherlands) aged 8 to 10 weeks were kept on a 12-hour day/night cycle. After 1 week accommodation, high-fat diet (HFD; total energy $22 \mathrm{MJ} / \mathrm{kg}, 60 \mathrm{~kJ} \%$ fat and $8.4 \mathrm{~kJ} \%$ sucrose; ssniff, Soest, Germany) or control diet (CD; total energy $15 \mathrm{MJ} / \mathrm{kg}, 11 \mathrm{~kJ} \%$ fat; ssniff) was fed for 10 weeks. ${ }^{10}$ Treatments were done from week 7 to 10 without dietary changes (Fig. 1A).

\section{Animal Exercising Protocol}

Exercise was performed using a special motor-driven mouse treadmill (Förderband GFB, Elmotec, Kleindöttingen, Switzerland; Fig. 1A) following a regimen of moderate exercise intensity. ${ }^{11,12}$ Animals were gradually accommodated to exercising for 1 week to reach the final speed of $650 \mathrm{~m} / \mathrm{h}$. Tired animals or animals not willing to follow the protocol were encouraged to continue running by gentle manual tips on their tail.

\section{Animal Surgery}

Isoflurane inhalation $(2 \%-4 \%)$ and subcutaneous buprenorphine application $(0.1 \mathrm{mg} / \mathrm{kg}$ bodyweight $)$ was used for all surgical interventions. For ischemia-reperfusion, 1-hour partial hepatic ischemia (68\%) was induced followed by analysis at 6 hours after

\section{Treatment protocol}

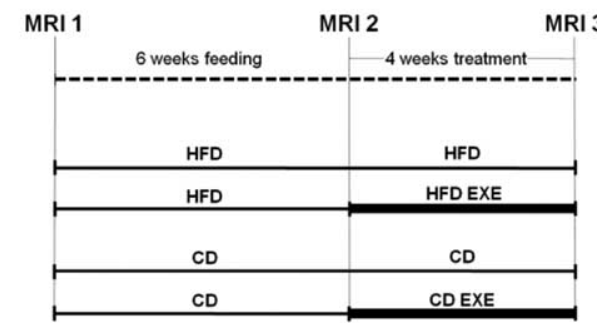

A
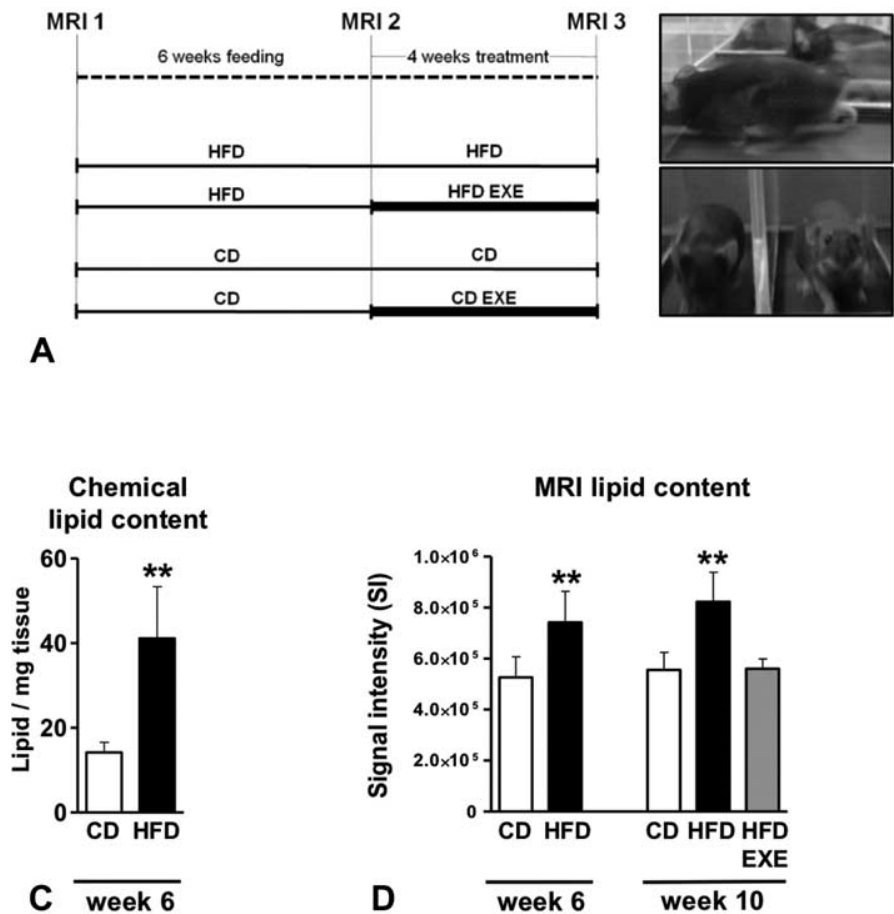

C $\overline{\text { week } 6}$

Serum

Insulin

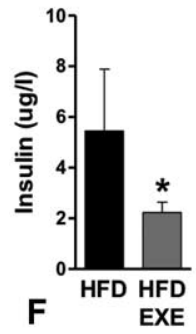

IPGTT

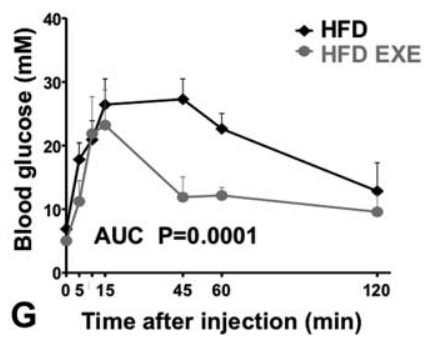

Liver/body weight changes

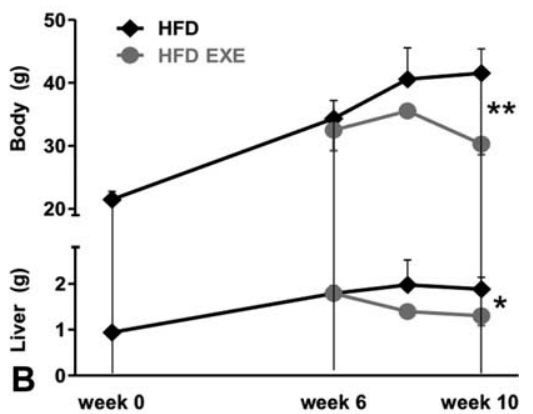

FIGURE 1. Exercising effects on metabolic parameters. A, Experimental setup. B, Body weight and liver weight changes during feeding and treatment periods. C, Chemical lipid content in liver after 6 weeks of control diet (CD) or HFD. D, MRI assessment of lipid content at 6 and 10 weeks. E, Histological steatosis at 10 weeks. F, Insulin levels at 10 weeks. G, Glucose tolerance at 10 weeks. $\mathrm{N}=5$ /group, Mann-Whitney (apart from panel G with AUC analysis), ${ }^{*} P<0.05,{ }^{* *} P<0.01$. 
reperfusion. Partial hepatectomies (68\% and extended 86\%) were performed as described. ${ }^{13}$

\section{AICAR Treatment}

The AMP analog 5-aminoimidazole-4-carboxamide ribonucleotide (AICAR) (Sigma, Buchs, Switzerland, \#A9978), known to activate AMPK, was injected i.p. in $100 \mu \mathrm{L}$ saline at $100 \mathrm{mg} / \mathrm{kg}$. ${ }^{14,15}$ Each AICAR application was timed to replace 1 exercising unit (5 doses/week for 4 weeks). I.p. saline injections served as control.

\section{MRI Protocol for Liver Fat Determination}

A small-animal 4.7-Tesla magnetic resonance imaging (MRI) scanner (Bruker BioSpin MRI, Inc., Billerica, Massachusetts, US) was used for in vivo monitoring of steatosis. Signal intensities (SI) were quantified in axial T2-weighted images using following parameters: repetition time: $2500 \mathrm{~ms}$; effective echo time: $45 \mathrm{~ms}$; flip angle: $180^{\circ}$; section thickness: $1 \mathrm{~mm}$; bandwidth $48.076 \mathrm{kHz}$; acquisition time: 10 minutes. Cross-sectional images containing liver parenchyma were analyzed using ParaVision 5.1 software (Billerica, Massachusetts, US). Mean SI were determined in regions of interest (ROI) covering all visible liver parenchyma.

\section{Chemical Liver Fat Measurement}

Lipid quantification in liver tissue was performed using a vanillin based method. ${ }^{16}$

\section{Serum Analyses}

Serum alanine transaminase (ALT) and aspartate transaminase (AST) levels were measured using a multiple biochemical analyzer (Dri-Chem 4000i, Fujifilm; Minato-ku, Tokyo, Japan). Serum HMGB1 and insulin levels were determined by enzyme linked immunosorbent assay (ELISA) (from Shino-Test, Hamburg, Germany, and from Mercodia, Uppsala, Sweden, respectively).

\section{Intraperitoneal Glucose Tolerance Test (IPGTT)}

After overnight fasting, D-glucose ( $2 \mathrm{~g} / \mathrm{kg}$, Merck, Darmstadt, Germany) was i.p. injected followed by a glycemic readout on tail vein blood (Glycemia reader Accu-Chek Aviva, Roche Diagnostics, Basel, Switzerland).

\section{Histological Analyses}

Archived liver sections $(3 \mu \mathrm{m})$ were stained with hematoxylin/ eosine or with antibodies listed in Supplementary Table 1, http:// links.lww.com/SLA/B448. ${ }^{13}$ Quantification was performed by manual counting 10 random visual fields in a blinded fashion. As a note of caution, visual field counting may differ from automated counting scores. For pAMPK and PPAR $\alpha$ scores, ImageJ software (Bethesda, Maryland, US) was used.

\section{Quantitative Real-Time Polymerase Chain Reaction (qPCR)}

RNA was extracted from 30 to $50 \mathrm{mg}$ of tissue using Trizol reagent (Invitrogen, Basel, Switzerland). qPCR was performed on cDNA (Thermo Script reverse transcription PCR System, Invitrogen) using an ABI Prism 7500 Sequence Detector system (PE Applied Biosystems, Rotkreuz, Switzerland). ${ }^{13}$ Results represent mean fold induction $\left(2^{-\Delta \mathrm{Ct}}\right) \pm \mathrm{SD}$ relative to the normalization control $18 \mathrm{~S}$ rRNA. TaqMan gene expression assays are listed in Supplementary Table 2, http://links.lww.com/SLA/B448.

\section{Hepatic ATP Content and ADP/ATP Ratio}

Frozen liver tissue was powdered, homogenized in HEPES buffer $\left(25 \mathrm{mmol} / \mathrm{L}\right.$ HEPES, $\left.10 \mathrm{mmol} / \mathrm{L} \mathrm{MgCl}_{2}, 0.02 \% \mathrm{NaAc}\right)$, and incubated for 10 minutes with $150 \mu \mathrm{L} 6 \%$ trichloroacetic acid
(Sigma, Buchs, Switzerland) and $100 \mu \mathrm{L}$ ATP releasing reagent (FLSAR; Sigma). The supernatant was analyzed in a luminometer (Labsystems Luminoscan 1.2-0; Labsystems, Helsinki, Finland) after addition of $50 \mu \mathrm{L}$ ATP monitoring reagent (Luciferase; Promega, Dübendorf, Switzerland). ATP concentrations were calculated from a calibration curve using pure ATP (disodium salt hydrate; Sigma) for each experiment.

ADP/ATP ratios were determined using a ratio assay kit (Abcam, Lucerne, Switzerland, \#ab65313) via bioluminescent detection.

\section{Statistical Analysis}

Variables are consistently presented as mean \pm SD from 5 animals/group. Differences between the respective experimental groups were assessed by Mann-Whitney $U$ testing. Area under the curve (AUC) comparisons were performed using 2-way ANOVA. $P$ $<0.05$ were considered significant and marked with stars $\left({ }^{*} P<0.05\right.$, $\left.{ }^{* *} P<0.01,{ }^{* * *} P<0.001\right)$. Statistical analyzes were performed using GraphPad Prism 6.0 (GraphPad Software, Inc., La Jolla, CA).

\section{RESULTS}

\section{Exercising Benefits in A Mouse Model Of Simple Steatosis}

Six weeks of feeding a HFD designed to mimic Western diet induced obesity and NAFLD without inflammation/fibrosis in mice. Mean body- and liver weight increased by $11.9 \mathrm{~g}( \pm 2.4)$ and $0.8 \mathrm{~g}$ $( \pm 0.2)$, respectively (Fig. 1B), accompanied by a marked elevation in hepatic lipid content (Fig. 1C). Mice with HFD-induced NAFLD were subjected to 4 weeks of 1 -hour-treadmill-running $(5 \times /$ week $)$ at approximately $70 \%$ of maximum aerobic capacity. ${ }^{11}$ HFD was maintained during the exercising period. After the exercising program, mice displayed significant reductions in body and liver weight relative to sedentary controls $(-10.3 \mathrm{~g}( \pm 4.5) ; P=0.008$ and $-0.6 \mathrm{~g}( \pm 0.4) ; P=$ 0.016 ; Fig. 1B). Given that steatosis increases with age, HFD animals were compared with age-matched mice on a $\mathrm{CD}$ that does not promote NAFLD (Fig. 1C/D). On MRI, exercise reduced lipid-associated signal intensities to those of CD mice (Fig. 1D), demonstrating it effectively negates the effects of the HFD. A similar fat reduction was evident on histology (Fig. 1E). In keeping with its antisteatotic effects, exercise also normalized hyperinsulinemia (Fig. 1F) and glucose tolerance (IPGTT, Fig. 1G) of HFD mice. The defattening properties of exercise were associated with the suppression of lipogenesis and the promotion of $\beta$-oxidation (Fig. 2A/B), consistent with a shift from fat storage to expenditure. A shift to lipid oxidation was noted already after two weeks of exercising (Supplementary Fig. 1, http://links.lww.com/ SLA/B448). A similar trend was noted in exercising CD mice (Supplementary Fig. 2, http://links.lww.com/SLA/B448), reflecting basic energy needs induced by exercise.

To confirm a promotion of energy production through exercise, we determined ATP content. Hepatic ATP levels were higher in HFD-EXE relative to HFD mice; on the other hand, the ADP/ATP ratio was increased through EXE (Fig. 2C), consistent with a higher energy usage. These findings imply exercise promotes in fatty liver the capacity for energy transfer and hence for recovery.

\section{The AMPK Activator AICAR Mimics the Beneficial Effects of Exercise on Hepatic Metabolism}

The energy sensor AMPK is activated by a relative paucity of ATP (ie, high AMP/ADP levels) and hence is induced through exercise. ${ }^{3}$ Accordingly, AMPK $\alpha$ gene expression (Prkaal, Fig. 3A) and active protein (phosphorylated at Thr172) were upregulated in liver of HFD-EXE relative to HFD mice (Fig. 3B, Supplementary Fig. 3, http://links.lww.com/SLA/B448). In support 
Lipogenesis

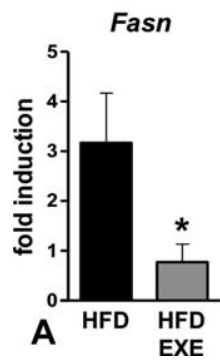

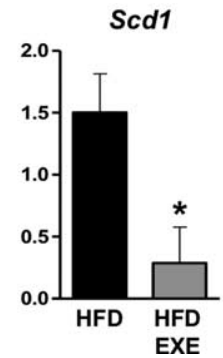

$\beta$-oxidation

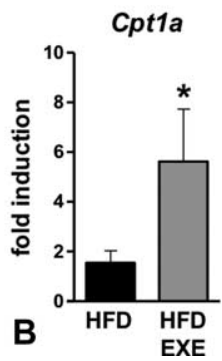

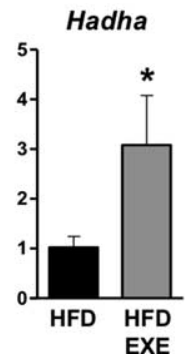

Energy status

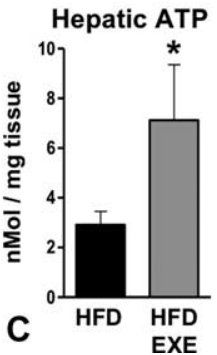

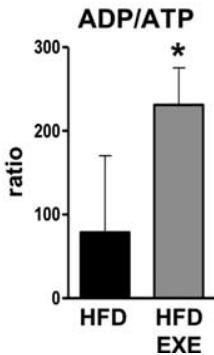

FIGURE 2. Exercise-induced energetic changes at 10 weeks of high fat diet (HFD). Hepatic expression of genes related to (A) lipogenesis and (B) beta-oxidation. C, Hepatic ATP content and ADP/ATP ratio. $N=5 /$ group, Mann-Whitney, ${ }^{*} P<0.05$.

of increased activity, PPAR $\alpha$ - a downstream target of AMPK and a transcription factor promoting lipid catabolism - was likewise elevated in HFD-EXE liver both at the mRNA and protein level (Fig. 3, Supplementary Fig. 4, http://links.lww.com/SLA/B448). To establish the reported antisteatotic effects of AMPK activation ${ }^{3}$ in our model, each exercising session was replaced by one bolus with the AMP analog AICAR, leading to comparable AMPK $\alpha$-PPAR $\alpha$ activation in liver (Fig. 4A, Supplementary Fig. 4, http://links.lww.com/SLA/B448). Saline i.p. injections for four weeks (ie, AICAR controls) did not affect body/liver weight of HFD mice (Fig. 4B). In contrast, a 4-week AICAR treatment reduced body/liver weight to that of HFD-EXE mice (Fig. 4C), along with congruent changes in histological steatosis (Fig. 4D), serum insulin, and glucose tolerance (Fig. 4E). Together, these findings confirm the antisteatotic/antidiabetic properties of AICAR and reveal that
AICAR has a potency to confer metabolic benefits on fatty liver akin to exercise. Therefore, we next tested the impact of exercise and AICAR on the capacity of HFD liver to sustain inflammatory stress or to recover from tissue loss.

\section{Exercise and AICAR Improve the Resistance of HFD Liver Towards Ischemic Insults}

Exposure of HFD mice to 1 hour of partial $(70 \%)$ hepatic ischemia induced major parenchymal injury as assessed by serum ALT, AST, and HMGB1 levels at 6 hours after reperfusion. The 4week-exercise program reduced injury after reperfusion on all parameters (Fig. 5A). AICAR treatment likewise had a protective effect, one that appeared pronounced compared with exercise (Fig. 5A). The protective effects of exercise/AICAR were mirrored in the extent of sinusoidal clotting (Fig. 5B), an important cause of
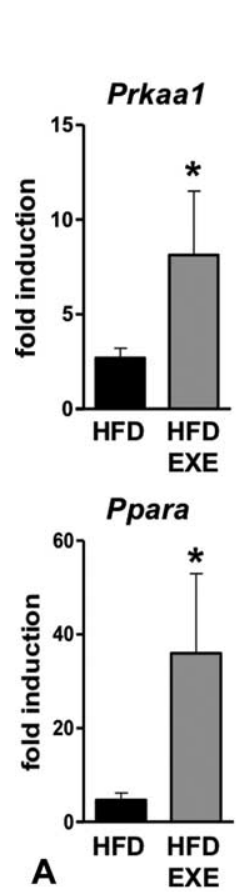
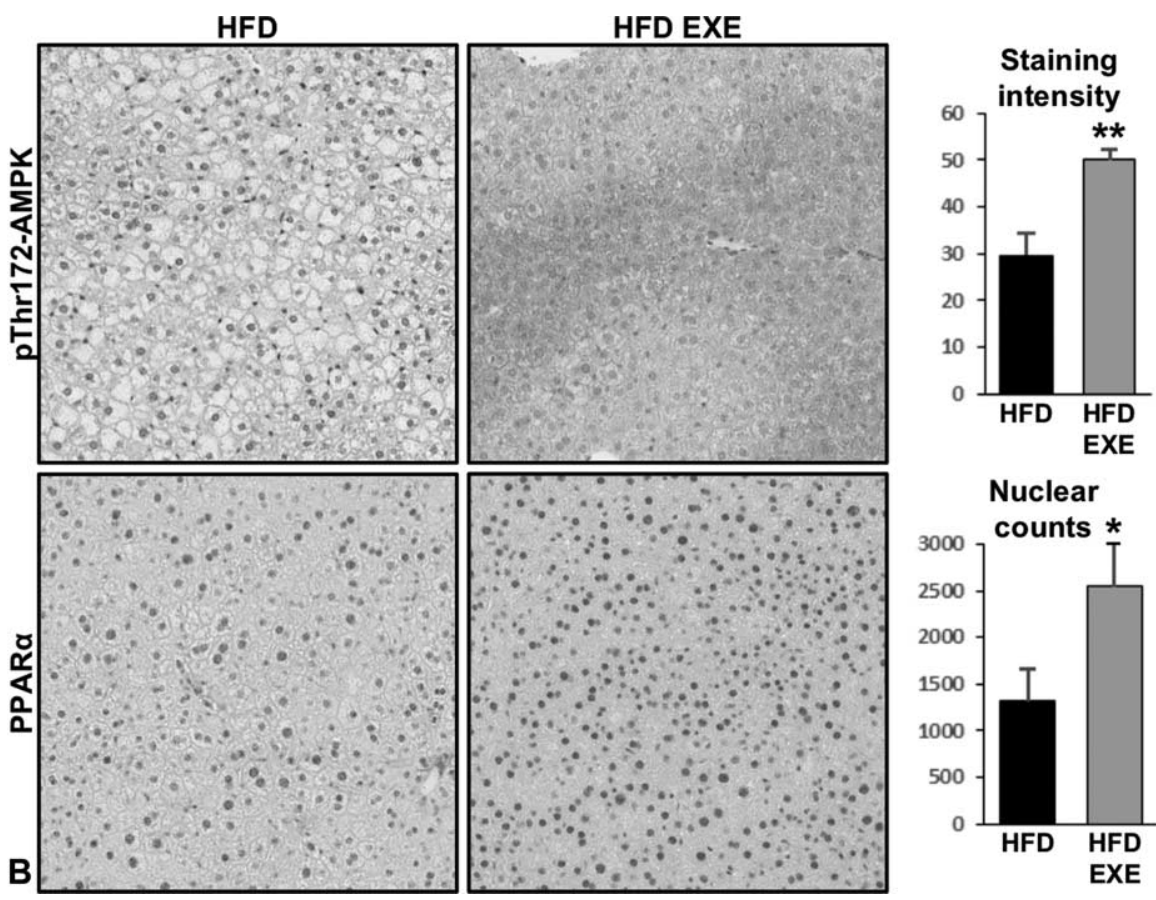

FIGURE 3. Exercise-associated AMPK activation at 10 weeks of high fat diet (HFD). A, Hepatic expression of genes encoding the subunit $\alpha 1$ of AMPK and its downstream target PPAR $\alpha$. B, Representative immunochemistry for activated pThr172-AMPK and PPAR $\alpha$ in HFD liver with or without exercise. Note the signal elevations for cytoplasmic pAMPK and nuclear PPAR $\alpha$ after exercise. Right graphs show computationally assessed staining scores. $N=5 /$ group, Mann Whitney, ${ }^{*} P<0.05$. 


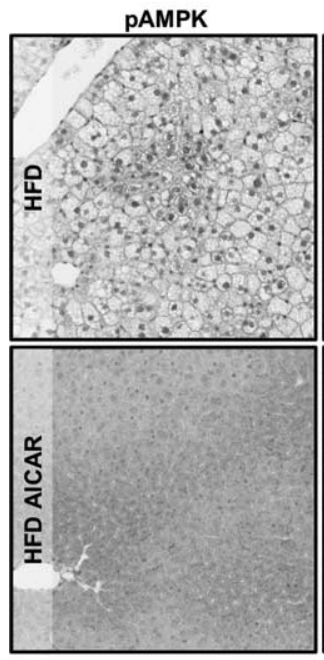

A

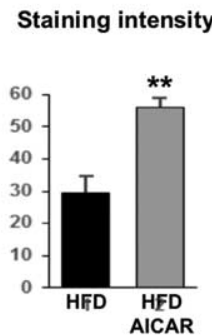

PPARa

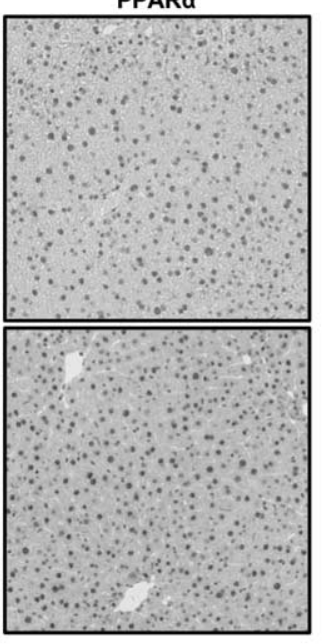

Nuclear counts

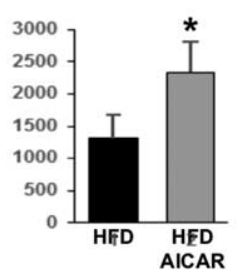

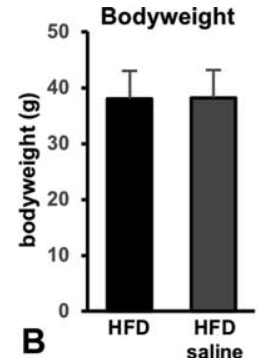

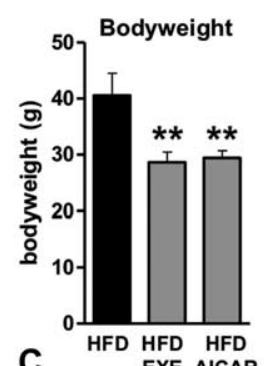

C

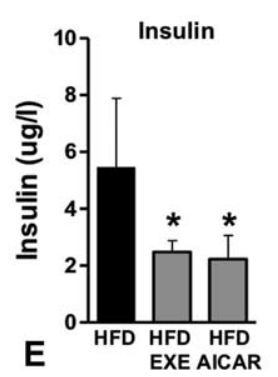

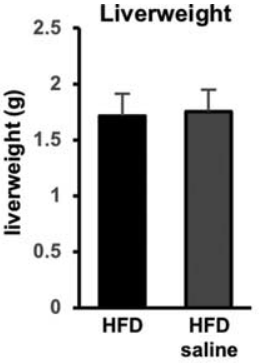
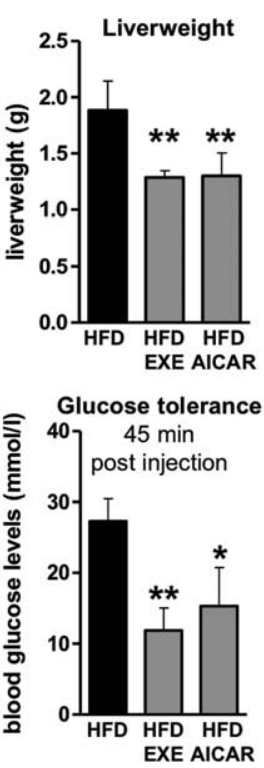

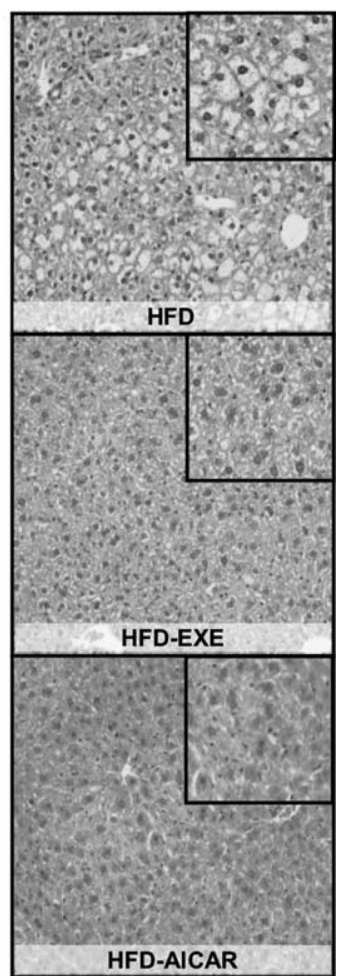

D

FIGURE 4. Metabolic changes at 10 weeks of high fat diet (HFD) after AICAR treatment relative to exercise. A, AMPK activation through AICAR as evinced by PAMPK and PPAR $\alpha$ immunochemistry. Representative images are shown. Graphs below show computationally assessed staining scores. B, Body/liver weight after 10 weeks of HFD alone or with a final 4-week i.p. saline (HFDsaline) treatment. C, Body and liver weight in HFD mice treated or not with exercise/AICAR. D, Representative images showing histological steatosis. $\mathrm{E}$, Serum insulin levels and glucose tolerance. $\mathrm{N}=5 /$ group; for panels $\mathrm{B} / \mathrm{C} / \mathrm{E}:$ Mann-Whitney, ${ }^{*} P<0.05,{ }^{* *} P<$ 0.01 .

impaired hepatic microcirculation after reperfusion. Therefore, exposure to exercise or AICAR markedly increases the tolerance of HFD liver towards ischemic insults.

\section{Exercise and AICAR Improve the Regenerative Capacity of HFD Liver}

To assess the effects of exercise/AICAR on liver regeneration, regenerative parameters were gathered at different time points after hepatectomy in HFD mice exposed to treatment or not. Given the different starting weight of the liver remnant at partial hepatectomy (ie, reduced steatosis after exercise/AICAR), liver weight gain was expressed as the percent increase over time. Compared with HFD mice, HFD-EXE mice displayed an accentuated weight regain, with the greatest increase (HFD-EXE 63\% \pm 7 vs HFD 51\% \pm 4 ) at 96 hours post resection (Fig. 6A). Ki67 nuclear counts were consistently increased in the HFD-EXE group, indicating more hepatocytes are in cycle (Fig. 6B). Likewise, total phosphohistone 3 ( $\mathrm{pH} 3$ ) counts, marking hepatocytes in the $\mathrm{G} 2$ and $M$ phases, were elevated from 48 hours (the usual peak of hepatocyte mitosis after hepatectomy ${ }^{13,17}$ ) onwards (Fig. 6C). To better appreciate the speed of cell cycle progression, we separately assessed bold $\mathrm{pH} 3$ positivity (marking M phase cells only ${ }^{13}$ and calculated the $\mathrm{M} /(\mathrm{G} 2+\mathrm{M})$ fraction as a measure of progression to mitosis. Exercise markedly elevated the $\mathrm{M} /(\mathrm{G} 2+\mathrm{M})$ fraction at 72 hours and 96 hours (Fig. 6D), indicating a prolonged and accelerated cell cycle progression, as reflected in the increased liver weight at 96 hours.

To determine whether AICAR has effects on regeneration similar to exercise, HFD-AICAR mice were analyzed at 96 hours after hepatectomy, when exercising effects were most obvious. AICAR treatment improved liver regeneration on all parameters assessed. Of note, the increases in percent liver weight, Ki67 counts, total $\mathrm{pH} 3$ counts and in the $\mathrm{M} /(\mathrm{G} 2+\mathrm{M})$ fraction were akin to those achieved through exercising (Fig. 6E). Apoptosis (cleaved caspase 3 stain, data not shown) was hardly detectable across samples, whereas hepatocyte death as assessed through serum ALT was reduced through exercise/AICAR (Fig. 6G).

To assess whether the proregenerative effects of exercise/ AICAR also translate into a regain of liver function after hepatectomy, we performed an $86 \%$-extended hepatectomy, ${ }^{13,17}$ which induces liver failure associated with a high mortality in steatotic mice. Both exercise and AICAR raised 10-day-survival after $86 \%$ hepatectomy (Fig. 6G), demonstrating their capacity to improve the functional recovery of HFD liver after extended tissue loss. 


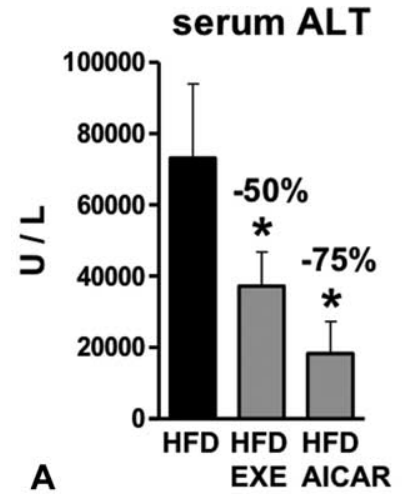

A

EXE AICAR

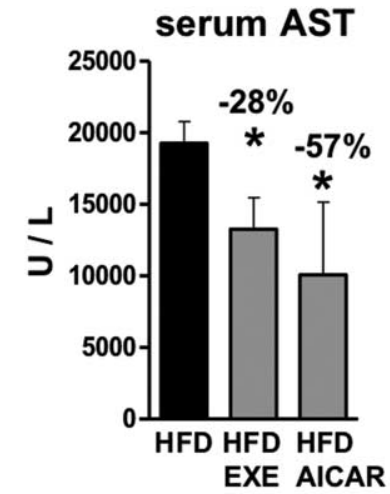

Histological necrosis

EXE

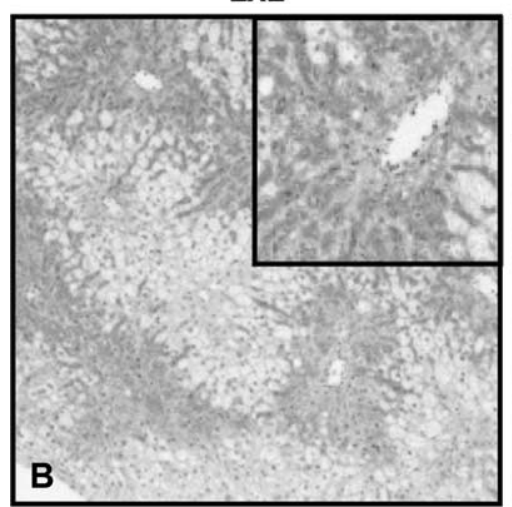

HFD EXE

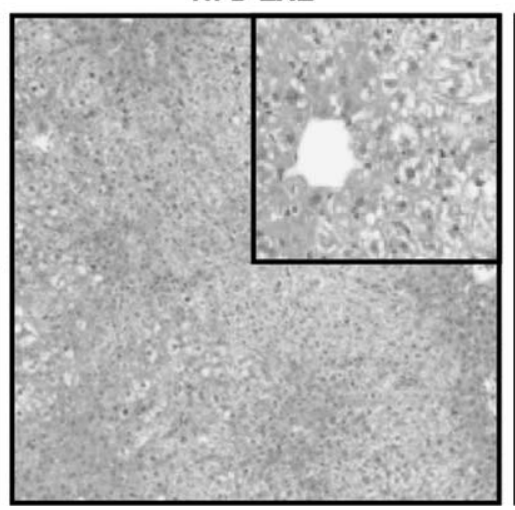

serum HMGB1

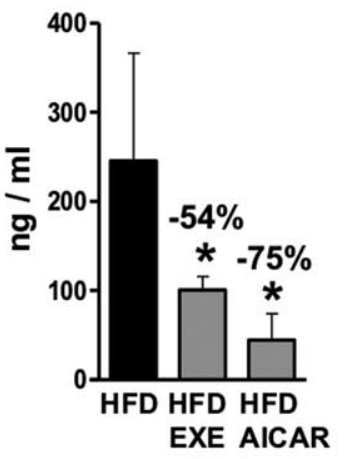

FIGURE 5. Impact of exercise/AICAR on ischemic tolerance of high fat diet (HFD) liver. A, Serum AST, ALT, and HMGB1 levels 6 hours after hepatic reperfusion in HFD, HFD-exercise (HFD-EXE), and HFD-5-aminoimidazole-4-carboxamide ribonucleotide (HFD-AICAR) mice. $\mathrm{N}=5$ /group, Mann-Whitney, ${ }^{*} P<0.05$. B, Representative images showing histological necrosis at 6 hours post reperfusion. Note the accentuated intrasinusoidal blood clot formation in livers of HFD mice. $\mathrm{N}=5 / \mathrm{group}$.

Together, the observations indicate that physical activity improves the capacity of HFD liver to regenerate, an effect that can likewise be achieved through AICAR.

\section{DISCUSSION}

Exercise and AICAR are known for their beneficial effects on fatty liver, in particular their antisteatotic properties and the associated improvements in insulin sensitivity and glucose handling. Here, we extend these benefits to two additional key aspects defining liver integrity that is the sensitivity to injury and the capacity to regenerate. Specifically, we show that exercise increases the tolerance of liver with diet-induced steatosis towards ischemic insults and enhances its regenerative response after tissue loss. More so, these benefits can be achieved by substituting training sessions with AICAR, suggesting pharmacological compounds may obviate the need for exercise to improve the function of fatty liver.

We established above findings in a mouse model of obesityassociated NAFLD, ${ }^{9}$ where exercise could replicate the known benefits of physical activity, including the ameliorations in body weight, steatosis, hyperinsulinemia, and glucose tolerance. ${ }^{2}$ Our observations are hence based on a valid experimental set up and underscore the importance of exercise for liver health. Moreover, our continuous model of diet-induced steatosis followed by treatment and then surgery offered the opportunity to test exercising and AICAR in settings relatively close to the clinic. Overall, the effects of the AMP analog AICAR were similar to exercising across the tested parameters, suggesting AICAR may be an effective exercise mimetic for fatty liver. Mechanistically, exercise activates various pathways, with AMPK clearly being a key target. ${ }^{2}$ Insofar, the action mechanisms of exercise and the AMPK activator AICAR may overlap, however AICAR also has AMPK-independent effects on hepatic metabolism. ${ }^{18}$ Nonetheless, the importance of AMPK activity for hepatometabolic health is recognized, and many of the benefits of exercising and AICAR are likely attributable to AMPK activation. $^{3}$

Conceivably, the reversal of steatosis is an important cause underlying the improvements in ischemic tolerance and regeneration, with exercise/AICAR simply reinstalling a normal capacity of liver to handle stress. However, exercise upregulated Prkaal/Ppara expression - along with congruent changes in metabolic genes (Supplementary Fig. 2, http://links.lww.com/SLA/B448) - and improved ischemic tolerance and regeneration also in lean liver, albeit not as consistent and to a lesser extent than in fatty liver. Similarly, short-term treatment with AICAR tended to mitigate ischemic injury and to promote regeneration in lean liver 

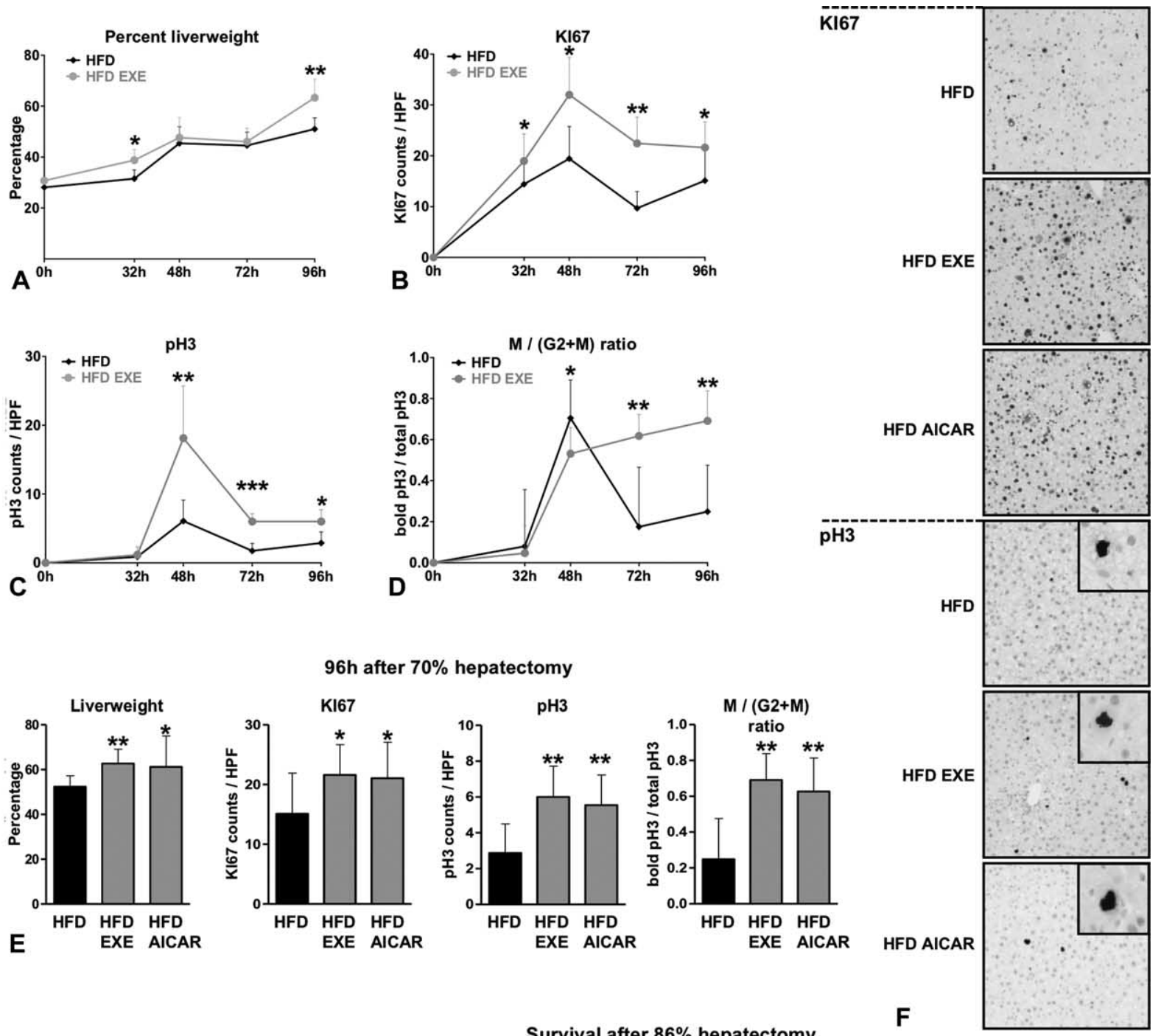

Survival after $86 \%$ hepatectomy

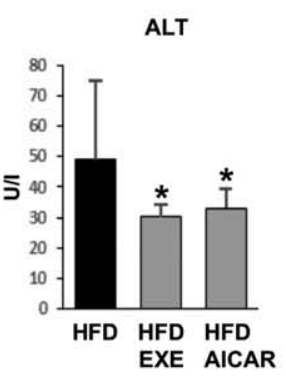

G
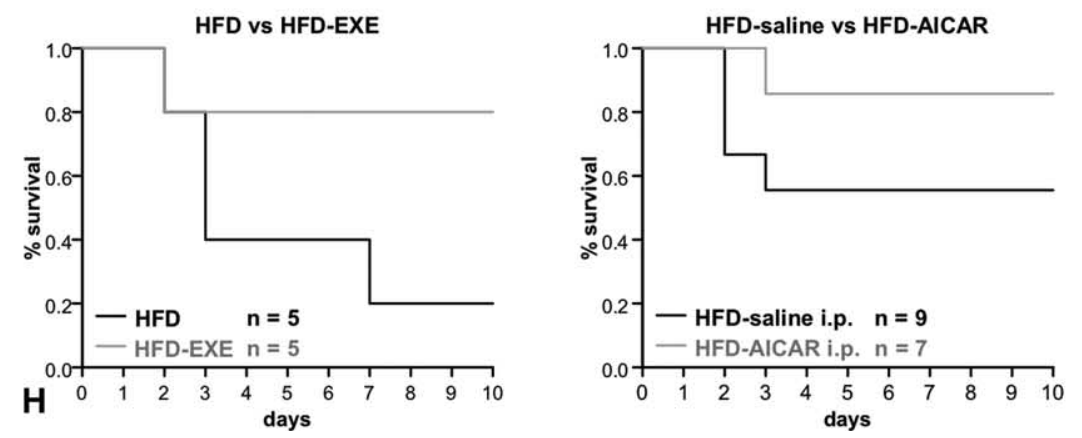

FIGURE 6. Impact of exercise/AICAR on regenerative capacity of high fat diet (HFD) liver. A, Percent liver weight, (B) Ki67 counts, (C) total $\mathrm{pH} 3$ counts, and (D) the $\mathrm{M} /(\mathrm{G} 2+\mathrm{M})$ fraction after partial heptatectomy on HFD and HFD-exercise (HFD-EXE) mice. E, Percent liver weight, Ki67 counts, pH3 counts, and the M/(G2+M) fraction at $96 \mathrm{~h}$ post partial heptatectomy in HFD versus HFD-EXE and HFD-5-aminoimidazole-4-carboxamide ribonucleotide (HFD-AICAR) mice. $\mathrm{N}=5 /$ group, Mann-Whitney, ${ }^{*} P<0.05,{ }^{* *} P<0.01$, $P<0.001$. F, Representative images showing Ki67 and $\mathrm{pH} 3$ stains. Insets show examples of bold pH3-positive hepatocytes undergoing mitosis. G, Serum ALT at 96 hours post partial heptatectomy. H, Kaplan-Meier survival estimates for HFD, HFD-EXE, HFD-saline, and HFD-AICAR mice after $86 \%$ hepatectomy. 
(Supplementary Figs. 5/6, http://links.lww.com/SLA/B448). Therefore, steatosis-independent effects seem to contribute to the benefits of exercise/AICAR for the liver.

The view that AMPK activation is key to the effects of exercise and AICAR is supported by the notion that 'preconditioning' - a strategy used in the clinic to protect liver from ischemia - seems to rely on AMPK activity irrespective of steatosis. ${ }^{19,20}$ Ischemia primarily damages the sinusoidal endothelium, however upon reperfusion, the burst in ROS leads to a self-perpetuating inflammatory response, whereby overactivated Kupffer cells attract neutrophils that eventually destroy the parenchyma. ${ }^{21,22}$ The increased sensitivity of steatotic liver likewise appears to root in the sinusoids, because the correction of fatrelated microcirculatory deficits normalizes its ischemic tolerance. ${ }^{23}$ Notably, AMPK activity can protect from endothelial dysfunction ${ }^{24}$ and skews macrophages to an anti-inflammatory M2 phenotype, ${ }^{25}$ a process that we have shown to shield against hepatic ischemia. ${ }^{22}$ Therefore, AMPK may counteract key events underlying the development of ischemic injury in both fatty and lean liver.

Furthermore, recovery from ischemia-reperfusion requires the regeneration of damaged parenchyma. Although unclarified, mechanistic overlap between regeneration after ischemic insults or after tissue loss is plausible. Therefore, exercise and AICAR may improve ischemic tolerance also through their proregenerative action. Notably, AMPK activity is known to contribute to regeneration. In vitro AMPK activation has been shown to promote hepatocyte proliferation by enhancing cyclin A and D expression. ${ }^{26,27}$ Conversely, constitutive deletion of Prkaal compromised cyclin A expression and cell cycle progression after hepatectomy. ${ }^{28}$ Besides the promotion of cyclins, other factors such as an improved energy provision ${ }^{29}$ are likely to add to the proregenerative effects of exercise or AICAR. Overall, the effects of AMPK activation on the liver are consistent with a central role of this molecule in the health effects of exercise/AICAR. In support of this notion, a 4-week AICAR treatment akin to this study improved endurance capacity of mice by $44 \%$ - remarkably in spite the animals being sedentary. ${ }^{30}$ A comprehensive assessment of exercise and AICAR in genetic loss/gain models would however be required to define the actual contribution of AMPK to their benefits.

The endemic raises in obesity and the metabolic syndrome, along with the associated cancer risks, call for effective treatments. ${ }^{31-33}$ Fatty liver is considered a key pathology behind the development of insulin resistance. ${ }^{34}$ Moreover, steatosis is an independent risk factor for liver cancer, ${ }^{35}$ with obese subjects having a close to 5-fold likelihood to die of hepatocellular carcinoma (HCC). The only accepted treatment for NAFLD is calorie restriction and physical activity. Exercising, however, is associated with compliance issues and can be difficult to implement, particularly in morbidly obese patients. Drugs mimicking the effects of exercise are hence desirable. Metformin, an indirect AMPK activator, is being considered a possible option, ${ }^{36}$ current evidence however is insufficient to recommend metformin against NAFLD.${ }^{37}$ Metformin primarily acts by decreasing mitochondrial capacity (leading to AMP accumulation activating AMPK) and therefore may not be an ideal exercise mimetic, as evinced through blunted exercise effects in prediabetic patients on metformin. ${ }^{38}$ AICAR, on the other hand, has been trialed particularly in myocardial ischemia, with some, but not all, studies documenting ischemic protection including a reduced mortality. ${ }^{39-41}$ Studies on AICAR and NAFLD are scarce, but AICAR did reduce hepatic glucose output along with reduced lipogenesis and increased $\beta$-oxidation in a small cohort of type-2-diabetic patients. ${ }^{42}$ Novel, allosteric AMPK activators are being developed and show promising hepatic effects in animal models of diabetes. ${ }^{43,44}$ Other classes (ie, not targeting AMPK) of 'exercise-mimetics' are also being evaluated in humans; at the current stage, it is unclear, which of these will remain clinically viable and some (such as the PPAR $\delta$ activator
GW1516) had to be abandoned because of unwanted effects, ${ }^{38,45}$ Importantly, single infusions of AICAR are considered as safe (based on data from $>4000$ patients, ${ }^{39-41}$ and multiple dosing likewise has been associated with an acceptable safety profile. ${ }^{46}$

Steatosis seems to particularly predispose to the development of non-cirrhotic $\mathrm{HCC}^{47}$ and thereby directly increases the demand for liver surgery. Surgery is the most indicated treatment for liver tumors; however, often is associated with ischemic periods and relies on regeneration. ${ }^{48}$ In addition to its ischemic and regenerative deficits, fatty liver also increases the risk for surgery-related liver failure (ie, small-for-size syndrome, SFSS). The SFSS can develop after extended hepatectomies leaving behind marginal remnants and represents the most frequent cause of death because of liver surgery. ${ }^{48}$ In our animal model, exercise and AICAR not only mitigated steatosis, but protected from ischemic injury, promoted regeneration, and improved survival after extended, SFSS-like resection. ${ }^{13,17}$ Notably, neither exercise ${ }^{2}$ nor pharmacological AMPK activators (ie, metformin ${ }^{49,50}$ ) seem to foster liver malignancy, suggesting their application may bear little oncological risks. Therefore, exercising programs or AICAR treatment may have potential to improve the outcomes of surgery for liver tumors.

In conclusion, our study highlights novel benefits of exercise and AICAR for liver health. Besides the mitigation of the metabolic deficiencies of fatty liver, exercise, and AICAR confer protection from ischemic injury and promote the recovery from tissue loss. These benefits are associated with AMPK activation, the target common to both exercise and AICAR. The relationship between steatosis and noncirrhotic HCC implies fatty liver is directly increasing the demand for surgery. Physical activity has the power to improve outcomes along the axis from steatosis to surgery; however, may fail owing to trivial reasons such as compliance. If similarly effective in man as in mice, AMPK promoters like AICAR may offer a simple solution to overcome some of the hazardous conveniences of our modern lifestyle.

\section{ACKNOWLEDGMENTS}

The authors would like to thank Eleonora Maurizio and Pia Fuchs for excellent technical assistance.

\section{REFERENCES}

1. Bauer UE, Briss PA, Goodman RA, et al. Prevention of chronic disease in the 21st century: elimination of the leading preventable causes of premature death and disability in the USA. Lancet. 2014;384:45-52.

2. Saran U, Humar B, Kolly P, et al. Hepatocellular carcinoma and lifestyles. J Hepatol. 2016;64:203-214.

3. Ruderman NB, Carling D, Prentki M, et al. AMPK, insulin resistance, and the metabolic syndrome. J Clin Invest. 2013;123:2764-2772.

4. Johnson NA, Sachinwalla T, Walton DW, et al. Aerobic exercise training reduces hepatic and visceral lipids in obese individuals without weight loss. Hepatology. 2009;50:1105-1112.

5. Hallsworth K, Fattakhova G, Hollingsworth KG, et al. Resistance exercise reduces liver fat and its mediators in non-alcoholic fatty liver disease independent of weight loss. Gut. 2011;60:1278-1283.

6. Church TS, Kuk JL, Ross R, et al. Association of cardiorespiratory fitness, body mass index, and waist circumference to nonalcoholic fatty liver disease. Gastroenterology. 2006;130:2023-2030.

7. Hannukainen JC, Nuutila P, Borra R, et al. Increased physical activity decreases hepatic free fatty acid uptake: a study in human monozygotic twins. J Physiol. 2007;578:347-358.

8. Rector RS, Uptergrove GM, Morris EM, et al. Daily exercise vs. caloric restriction for prevention of nonalcoholic fatty liver disease in the OLETF rat model. Am J Physiol Gastrointest Liver Physiol. 2011;300:G874-G883.

9. Linecker M, Limani P, Kambakamba P, et al. Omega-3 fatty acids protect fatty and lean mouse livers after major hepatectomy. Ann Surg. 2017;266:324-332.

10. Marsman HA, Heger M, Kloek JJ, et al. Omega-3 fatty acids reduce hepatic steatosis and consequently attenuate ischemia-reperfusion injury following partial hepatectomy in rats. Dig Liver Dis. 2011;43:984-990. 
11. Schefer V, Talan MI. Oxygen consumption in adult and AGED C57BL/6J mice during acute treadmill exercise of different intensity. Exp Gerontol. 1996;31:387-392.

12. Piguet AC, Saran U, Simillion C, et al. Regular exercise decreases liver tumors development in hepatocyte-specific PTEN-deficient mice independently of steatosis. J Hepatol. 2015;62:1296-1303.

13. Lehmann K, Tschuor C, Rickenbacher A, et al. Liver failure after extended hepatectomy in mice is mediated by a p21-dependent barrier to liver regeneration. Gastroenterology. 2012;143:1609-1619.

14. Gaskin FS, Kamada K, Yusof M, et al. AICAR preconditioning prevents postischemic leukocyte rolling and adhesion: role of K(ATP) channels and heme oxygenase. Microcirculation. 2009;16:167-176.

15. Escobar DA, Botero-Quintero AM, Kautza BC, et al. Adenosine monophosphate-activated protein kinase activation protects against sepsis-induced organ injury and inflammation. J Surg Res. 2015;194:262-272.

16. Van Handel E. Rapid determination of total lipids in mosquitoes. J Am Mosq Control Assoc. 1985;1:302-304.

17. Tschuor C, Kachaylo E, Limani P, et al. Constitutive androstane receptor (Car)-driven regeneration protects liver from failure following tissue loss. $J$ Hepatol. 2016;65:66-74.

18. Guigas B, Taleux N, Foretz M, et al. AMP-activated protein kinase-independent inhibition of hepatic mitochondrial oxidative phosphorylation by AICA riboside. Biochem J. 2007;404:499-507.

19. Peralta C, Bartrons RA, Serafin A, et al. Adenosine monophosphate-activated protein kinase mediates the protective effects of ischemic preconditioning on hepatic ischemia-reperfusion injury in the rat. Hepatology. 2001;34:1164-1173.

20. Carrasco-Chaumel E, Rosello-Catafau J, Bartrons R, et al. Adenosine monophosphate-activated protein kinase and nitric oxide in rat steatotic liver transplantation. J Hepatol. 2005;43:997-1006.

21. Peralta C, Jimenez-Castro MB, Gracia-Sancho J. Hepatic ischemia and reperfusion injury: effects on the liver sinusoidal milieu. J Hepatol. 2013;59:1094-1106.

22. Raptis DA, Limani P, Jang JH, et al. GPR120 on Kupffer cells mediates hepatoprotective effects of omega 3-fatty acids. J Hepatol. 2014;60:625-632.

23. El-Badry AM, Jang JH, Elsherbiny A, et al. Chemical composition of hepatic lipids mediates reperfusion injury of the macrosteatotic mouse liver through thromboxane A(2). J Hepatol. 2011;55:1291-1299.

24. Zhang WC, Wang QL, Wu Y, et al. Endothelial cell-specific liver kinase B1 deletion causes endothelial dysfunction and hypertension in mice in vivo. Circulation. 2014;129:1428-1439.

25. Mounier R, Theret M, Arnold L, et al. AMPK alpha 1 regulates macrophage skewing at the time of resolution of inflammation during skeletal muscle regeneration. Cell Metabol. 2013;18:251-264

26. Martinez-Chantar ML, Vazquez-Chantada M, Garnacho M, et al. S-adenosylmethionine regulates cytoplasmic HuR via AMP-activated kinase. Gastroenterology. 2006;131:223-232.

27. Varela-Rey M, Beraza N, Lu SC, et al. Role of AMP-activated protein kinase in the control of hepatocyte priming and proliferation during liver regeneration. Exp Biol Med. 2011;236:402-408.

28. Merlen G, Gentric G, Celton-Morizur S, et al. AMPK alpha 1 controls hepatocyte proliferation independently of energy balance by regulating Cyclin A2 expression. J Hepatol. 2014;60:152-159.

29. Kachaylo E, Tschuor C, Calo N, et al. PTEN down-regulation promotes betaoxidation to fuel hypertrophic liver growth after hepatectomy in mice. Hepatology. 2017;66:908-921.

30. Narkar VA, Downes M, Yu RT, et al. AMPK and PPARdelta agonists are exercise mimetics. Cell. 2008;134:405-415.

31. Malik VS, Willett WC, Hu FB. Global obesity: trends, risk factors and policy implications. Nat Rev Endocrinol. 2013;9:13-27.
32. Smyth S, Heron A. Diabetes and obesity: the twin epidemics. Nat Med. 2006; $12: 75-80$

33. Vos T, Barber RM, Bell B, et al. Global, regional, and national incidence, prevalence, and years lived with disability for 301 acute and chronic diseases and injuries in 188 countries, 1990-2013: a systematic analysis for the Global Burden of Disease Study 2013. Lancet. 2015;386:743-800.

34. Humar B, Semmo N. Endocannabinoid signaling in liver pathologies. In: Dufour F, Clavien A, eds. Signaling Pathways in Liver Diseases. 3rd ed., Oxford: Wiley Blackwell; 2015:226-239.

35. Kurosaki M, Hosokawa T, Matsunaga K, et al. Hepatic steatosis in chronic hepatitis $\mathrm{C}$ is a significant risk factor for developing hepatocellular carcinoma independent of age, sex, obesity, fibrosis stage and response to interferon therapy. Hepatol Res. 2010;40:870-877.

36. Saenz A, Fernandez-Esteban I, Mataix A, et al. Metformin monotherapy for type 2 diabetes mellitus. Coch Database System Rev. 2005;20:CD002966.

37. Mills EP, Brown KPD, Smith JD, et al. Treating nonalcoholic fatty liver disease in patients with type 2 diabetes mellitus: a review of efficacy and safety. Ther Adv Endocrinol Metab. 2018;9:15-28.

38. Li S, Laher I. Exercise pills: at the starting line. Trends Pharmacol Sci. 2015;36:906-917.

39. Mangano DT. Effects of acadesine on myocardial infarction, stroke, and death following surgery. A meta-analysis of the 5 international randomized trials. The Multicenter Study of Perioperative Ischemia (McSPI) Research Group. JAMA. 1997;277:325-332.

40. Mangano DT, Miao Y, Tudor IC, et al. Post-reperfusion myocardial infarction: long-term survival improvement using adenosine regulation with acadesine. $J$ Am Coll Cardiol. 2006;48:206-214.

41. Newman MF, Ferguson TB, White JA, et al. Effect of adenosine-regulating agent acadesine on morbidity and mortality associated with coronary artery bypass grafting: the RED-CABG randomized controlled trial. JAMA. 2012;308:157-164.

42. Boon H, Bosselaar M, Praet SF, et al. Intravenous AICAR administration reduces hepatic glucose output and inhibits whole body lipolysis in type 2 diabetic patients. Diabetologia. 2008;51:1893-1900.

43. Li YY, Yu LF, Zhang LN, et al. Novel small-molecule AMPK activator orally exerts beneficial effects on diabetic db/db mice. Toxicol Appl Pharmacol. 2013;273:325-334.

44. Wu L, Zhang L, Li B, et al. AMP-activated protein kinase (AMPK) regulates energy metabolism through modulating thermogenesis in adipose tissue. Front Physiol. 2018;9:122.

45. Handschin C. Caloric restriction and exercise "mimetics": Ready for prime time? Pharmacol Res. 2016;103:158-166.

46. Van Den Neste E, Cazin B, Janssens A, et al. Acadesine for patients with relapsed/refractory chronic lymphocytic leukemia (CLL): a multicenter phase I/II study. Cancer Chemother Pharmacol. 2013;71:581-591.

47. van Meer S, van Erpecum KJ, Sprengers D, et al. Hepatocellular carcinoma in noncirrhotic livers is associated with steatosis rather than steatohepatitis: potential implications for pathogenesis. Eur $J$ Gastroenterol Hepatol. 2016;28:955-962.

48. Clavien P, Petrowsky H, DeOliveira ML, et al. Medical progress: strategies for safer liver surgery and partial liver transplantation. New Eng J Med. 2007;356:1545-1559.

49. Ma SJZY, Zhou PC, Xiao YN, et al. Metformin use improves survival of diabetic liver cancer patients: systematic review and meta-analysis. Oncotarget. 2016;7:66202-66211.

50. He XK, Su TT, Si JM, et al. Metformin is associated with slightly reduced risk of colorectal cancer and moderate survival benefits in diabetes mellitus: a meta-analysis. Medicine. 2016;95:e2749. 